\title{
BISp Informationen
}

Ger J Exerc Sport Res 2020 · 50:331-332

https://doi.org/10.1007/s12662-020-00657-8

(c) Springer-Verlag GmbH Deutschland, ein Teil von Springer Nature 2020

Bundesinstitut

für Sportwissenschaft
Bundesinstitut für Sportwissenschaft (BISp)

Marcus Punte

Fachgebiet Innovationsmanagement

Graurheindorfer Str. 198

D-53117 Bonn

marcus.punte@bisp.de

www.bisp.de

\section{Sportsatellitenkonto (SSK): Bundesministerium für Wirt- schaft und Energie und Bun- desinstitut für Sportwissen- schaft veröffentlichen neuen Themenbericht}

\begin{abstract}
Das Bundesministerium für Wirtschaft und Energie (BMWi) und das Bundesinstitut für Sportwissenschaft (BISp) veröffentlichen regelmäßig neue Daten zur deutschen Sportwirtschaft.

Auf Basis der amtlichen Statistikdaten, ergänzt um umfangreiche Ergebnisse aus zusätzlichen Primärerhebungen, werten die Gesellschaft für Wirtschaftliche Strukturforschung (GWS, Osnabrück) und die 2HMforum. $\mathrm{GmbH}$ (Mainz) die Ergebnisse zur Bedeutung der Querschnittsbranche Sportwirtschaft aus. Der diesjährige sog. Themenbericht befasst sich mit dem „Beitrag des Sports zur Erfüllung der WHOEmpfehlungen für körperliche Aktivität“.
\end{abstract}

Bewegungsmangel hat nicht nur negative gesundheitliche Folgen für den Einzelnen. Körperliche Inaktivität verursacht auch direkte und indirekte volkswirtschaftliche Kosten. Die Weltgesundheitsorganisation (WHO) hat Bewegungsempfehlungen veröffentlicht, deren Einhaltung das Risiko für das Auftreten einer Vielzahl von Er- krankungen verringern kann. Auf Basis des Sportsatellitenkontos wird untersucht, welchen Beitrag der Sport zur Erfüllung der WHO-Empfehlungen leistet. In der erwachsenen Bevölkerung erfüllen $34 \%$ die WHOEmpfehlungen allein durch ihre sportliche Aktivität. Insgesamt trägt der Sport rund 75 \% zur Erfüllung bei, wobei sich deutliche Unterschiede zwischen verschiedenen Bevölkerungsgruppen sowie zwischen den einzelnen Sportarten zeigen.

Das eBook mit den Zahlen und Hintergründe finden Sie unter dem folgenden Link:

http:// my.page 2 flip.de/ 15646901/18757231/18761118/ html5.html\#/1

Die Studie ist auch in einer englischen Version unter diesem Link verfügbar:

ht t p : / / my.page $2 \mathrm{flip}$. $\mathrm{de} / 15646901 / 18757381 /$ 18761121/html5.html\#/1

Weitere Informationen und Veröffentlichungen zum Projekt „Monitoring Sportsatellitenkonto " finden Sie unter: www.sportsatellitenkonto.de

\section{REGman-Praxisworkshop}

Gemeinsam mit der REGmanProjektgruppe lud das Bundesinstitut für Sportwissenschaft (BISp) zum 2. REGman-Praxisworkshop im Februar 2020 nach Mainz ein. Ziel der Veranstaltung war es, den Transfer von wissenschaftlichen Erkenntnissen in die Sportpraxis und den gegenseitigen Austausch zwischen allen Beteiligten anzuregen und zu unterstützen.

Die Veranstaltung richtete sich mit praxisnahen Vorträgen und anwendungsorientierten Sessions insbesondere an Vertreterinnen und Vertreter der Sportpraxis. Mehr als 100 Gäste folgten der Einladung und hörten am ersten Workshop-Tag spannende Vorträge zu Themen wie „Zur Bedeutung eines individualisierten Regenerationsmanagements“, „Regenerationsmanagement durch Athletenmonitoring " oder „Regenerationsbedürfnisse und Regenerationsmanagement in ausgewählten Sportarten (Eishockey, Tennis, Hallen-Volleyball)“. Abgerundet wurde der erste Tag mit einer Podiumsrunde, in der die beiden Spitzensportlerinnen, Diana Sujew und Sabrina Mockenhaupt, aus ihrem persönlichen Erfahrungsschatz rund um das Thema Leistungssport und Regeneration berichteten.

Am zweiten Tag hatten die Teilnehmerinnen und Teilnehmer dann die Möglichkeit, die Projektergebnisse in vier Praxisworkshops direkt auszuprobieren und anzuwenden. Somit konnten alle Teilnehmerinnen und Teil- nehmer die für sich und ihre bzw. seine Sportart passenden spezifischen Elemente direkt mit in den Praxisalltag übernehmen.

Der 2. Praxisworkshop und die rege Teilnahme aus der Sportpraxis zeigt, dass die Ausrichtung der REGman-Projektkonzeption in der zweiten Phase (2017-2020) unmittelbar an den Bedarfen der Sportpraxis einen großen Mehrwert hervorgebracht hat. Schnelle und effektive Regeneration wird im Spitzensport angesichts ausufernder Wettkampfkalender und hoher Trainingsbelastungen immer wichtiger. Gleichzeitig besteht ein Defizit an wissenschaftlich fundierten Empfehlungen, nach denen sich die Trainingspraxis richten kann. In Anbetracht dieses Unterstützungsbedarfes fördert das Bundesinstitut für Sportwissenschaft seit 2012 das Projekt „Optimierung von Training und Wettkampf: Regenerationsmanagement im Spitzensport" (REGman). REGman ist Bestandteil des Forschungsprogramms für das Wissenschaftliche Verbundsystem im Leistungssport.

Die folgenden Links bieten Ihnen weiterführende Informationen zum Projekt sowie zum 2. REGman-Praxisworkshop:

Projektwebsite: https://regman.org/

Publikation mit aktuellen Forschungsergebnisse und Handlungsempfehlungen: ht tp:// my.page 2 flip.de/ 15646901/18599754/18602187/html5.html\#/1 
Aufzeichnung des 2. Praxisworkshops sowie REGman-spezifische Videos aus dem BISpVideowettbewerb finden Sie auf dem YouTube-Kanal des BISp: https://www.youtube.com/ channel/UCvElxVwYnDslBBaMJ3hNsoQ.

\section{Sportgroßveranstaltungen für Deutschland!? Eine Sonderpublikation}

Sollte sich Deutschland um Sportgroßveranstaltungen bewerben? In einer Sonderpublikation hat das BISp unterschiedliche Perspektiven $\mathrm{zu}$ diesem Fragenkomplex veröffentlicht.

Hintergrund ist der Arbeitsauftrag, den sich die Bundesregierung im Koalitionsvertrag (2018) erteilt hat: „Der Aufbau und die Umsetzung einer langfristig angelegten Strategie für Sportgroßveranstaltungen, insbesondere olympische und paralympische Spiele sowie Universiaden, wird die Spitzensportreform begleiten. Hierfür wollen wir übergeordnete Leitlinien entwickeln.“ Ein erstes Grobkonzept haben das Bundesministerium des Innern, für Bau und Heimat (BMI) und der Deutsche Olympische Sportbund (DOSB) im Februar 2020 vorgelegt.

Zur Unterstützung dieser Maßnahme hat das Bundesinstitut für Sportwissenschaft einen zweibändigen Überblick zusammengestellt, für den die

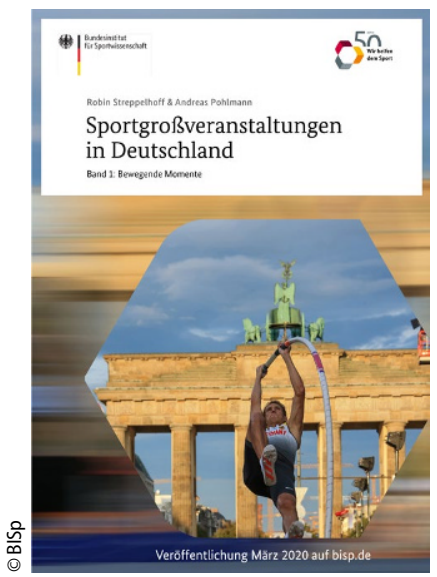

in Deutschland führenden Wissenschaftlerinnen und Wissenschaftler sowie einige Protagonisten aus Politik, Sport und Wirtschaft für Kurzbeiträge gewonnen wurden. Ziel war es, die Chancen und Risiken für die Ausrichtung entsprechender Veranstaltungen aus den Perspektiven der Gesellschaft, Wirtschaft, Politik und des Sports zu skizzieren. Band I liegt nun vor.

In seinem Geleitwort betont der DOSB die sportpolitisch hohe Relevanz des Sammelbandes: „Die Chancen von Sportgroßereignissen, ihre gesellschaftliche Kraft und auch die Vernetzung in der globalisierten Welt in die Debatte einzubringen, ist notwendig, damit wir auch in Zukunft kollektive Sport-Erinnerungen und damit neue Spiegelbilder unserer Gesellschaft formen können. Der hier vorliegende Sammelband bietet hierzu vielfältige Ansatzpunkte."

Und auch das BMI hebt die Beratungsleistung durch das BISp hervor: „Dieser Sammelband bietet eine geeignete Plattform für den Austausch unterschiedlicher Ansichten und Meinungen und ist damit ein wichtiger Bestandteil der Diskussion $\mathrm{zu}$ Ausgestaltung und Umsetzung der Nationalen Strategie für Sportgroßveranstaltungen.“

Zum eBook bzw. kostenfreien Download von Sportgroßveranstaltungen in Deutschland, Band I kommen Sie über den folgenden Link: h t t p: / / my.page 2 flip.de/ 15646901/18599344/18601946/ html5.html\#/1

\section{Grußwort des BISp}

Die Entwicklungen rund um das Coronavirus stellen uns alle vor große Herausforderungen. Wir wünschen Ihnen alles Gute und vor allem Gesundheit in dieser etwas anderen Zeit.

Ihr BISp-Team 